\title{
Ambiente, valores y percepción del futuro de los niños, niñas y adolescentes en situación de calle en la provincia de Lima
}

\author{
Scientific Rigor Level of Master's Thesis in Education - Case Study: Public \\ University
}

Mariana Benavente Flores ${ }^{1}$

\section{RESUMEN}

El artículo resume los principales hallazgos de una investigación exploratoria (cualitativa y cuantitativa) acerca de los niños, niñas y adolescentes en situación de calle en la Provincia de Lima, Perú. Esta investigación tuvo como objetivo conocer los ambientes (familiar y escolar) en los cuales niños, niñas y adolescentes en situación de calle se encontraban antes de acudir al espacio callejero. Asimismo, se estudió la realidad a la cual niños, niñas y adolescentes están expuestos una vez que inician el vínculo con la calle. La investigación finaliza con sus expectativas acerca del futuro, que lejos de ser fatalistas, están llenas de esperanza y el deseo de reivindicarse.

\section{Palabras clave}

Niños en situación de calle, familias expulsoras, rehabilitación social. Niños y adolescentes en situación de calle, familias expulsoras, colegios desintegradores.

\section{ABSTRACT}

This paper summarizes the main findings of an exploratory research (qualitative and quantitative) on street children and adolescents in the Province of Lima, Peru. This research aimed to have deeper knowledge about the environments (family and school) of children and adolescents before they started to live on the streets. Moreover, this research explored the reality that children and teenagers face once they start to live on the streets. This research also comprised the children's expectations about the future, which, far from being fatalistic, were full of hope and desire for vindication.

\section{Keywords}

Street children, expelling families, social rehabilitation. street children and teenagers, rejection by familis, disintegration in schools.

\section{Introducción}

De acuerdo con Yachay, programa nacional para la infancia en situación de calle del Ministerio de la Mujery Poblaciones Vulnerables (MIMP), en el Perú existen cuatro perfiles de niños y adolescentes en situación de calle: niños, niñas y adolescentes que trabajan en la calle; niños, niñas y adolescentes en situación de mendicidad; niños niñas y adolescentes que viven en la calle; $y$, niños, niñas y adolescentes en situación de explotación sexual infantil.
Una de las pocas publicaciones acerca de esta temática ("Perfil de niños, niñas y adolescentes en situación de calle" de Voces para Latinoamérica y Sinergia por la Infancia), señala que los niños y adolescentes que empiezan a frecuentar la calle pasan por distintas etapas hasta romper el vínculo familiar, muchos de ellos generan dependencia a las drogas. (Voces para Latinoamérica, 2008)

A pesar de tratarse de un grave problema que va en contra de lo estipulado en nuestra

Bachiller en Comunicaciones por Universidad san Ignacio de Loyola (USIL), encargada del área de Investigación y Comunicación para el Desarrollo en Vox Populi Consultoría, autora del libro de investigación "Los niños de la calle". 
legislación con relación a la infancia (en el Código de los niños y adolescentes), no existen datos actuales acerca de la población de menores de edad en situación de calle que permitan establecer objetivos claros para abordar el problema.

De los perfiles establecidos por Yachay, la información es escasa y no existen datos estadísticos excepto el caso de niños $y$ adolescentes en situación de trabajo en calle, quienes suman 231,286 niños y adolescentes que laboran en dicho espacio (Yachay, sf, sp).

Debido a la insuficiente información acerca de este problema, esta investigación significa una primera aproximación a la población de menores de edad en situación de calle. Se trata de un estudio exploratorio que busca conocer quiénes son los niños y adolescentes en situación de calle, cómo llegaron a este espacio, qué es lo que pasa en la calle y cuáles son las expectativas que tienen acerca de su futuro.

Los resultados de este estudio pretenden ser una base para hacer futuras investigaciones con respecto al tema, por ejemplo, enfocadas en el pandillaje, eje de organización social presente en las calles, que canaliza las necesidades afectivas y económicas de niños y adolescentes provenientes de familias expulsoras.

\section{Metodología}

La investigación consistió en un estudio cuantitativo y cualitativo con el objetivo de conocer el ambiente, los valores y la percepción del futuro de niños y adolescentes en situación de calle. Fue llevada a cabo por la empresa de investigación Vox Populi.

Para la investigación cuantitativa se utilizó como herramienta la encuesta (estructurada), que fue aplicada en una muestra de la población de estudio. Se tomó como universo a los niños y adolescentes en situación de calle $y$ en proceso de rehabilitación social (antes en situación de calle), en la provincia de Lima, entre los 6 y 17 años.

La muestra fue de 237 niños, niñas $y$ adolescentes en la provincia de Lima, 120 de ellos en situación de calle y 137 en proceso de rehabilitación. Los segmentos considerados fueron los siguientes: edad (niños entre 6 y 11 años, $y$, adolescentes entre 12 y 17 años), género (hombres y mujeres) y condición (en situación de calle y en proceso de rehabilitación).

Para la muestra de menores de edad en situación de calle se identificaron puntos de muestreo en distintos distritos de Lima. Con respecto a la muestra de niños y adolescentes en proceso de rehabilitación, los cuestionarios fueron aplicados en seis instituciones: Casa hogar Juan Pablo II, Casa hogar Los Delfines (CEDRO), Casa hogar Santa María (CEDRO), Casa Hogar Luzmila Maqueira, Centro de Integración para Menores en Abandono (CIMA) e Instituto Mundo Libre.

Con relación a la investigación cualitativa, se utilizaron tres herramientas: la entrevista en profundidad, el focus group o grupo focal y el test proyectivo. En este caso, el universo estuvo conformado por adolescentes en proceso de rehabilitación entre los 12 y 17 años, que antes estuvieron en situación de calle. El trabajo de campo para la investigación cualitativa se realizó en CIMA y el Instituto Mundo Libre.

Asimismo, los resultados de la investigación se complementaron con entrevistas en profundidad a especialistas. Se entrevistó a Jorge Saavedra (director de CIMA), Betty Godoy (psicóloga en CIMA), Alcida Cabrera (asistenta social en Juan Pablo II), Francisco Iturrino (director en Juan Pablo II), Manoel Torquato (presidente de la Campaña Nacional Crianza Nao e de Rua en Brasil), Flavia Centurión (psicóloga) y Jorge Valencia (abogado especialista en temas de familia e infancia).

\section{Resultados}

Los resultados de esta investigación están divididosencincotemasprincipales, losprimeros dos (familia y colegio), son los espacios en los cuales los niños y adolescentes se encontraban antes de acudir al espacio callejero. El tercer aspecto, la calle; que comprende la realidad de los niños y adolescentes en situación de calle y cómo algunos factores influyen en el debilitamiento del vínculo familiar. Los 
resultados de la investigación finalizan con el sentido de pertenencia (con respecto al Perú) y las expectativas de futuro, que son positivos a pesar de las situaciones tan negativas por las cuales han pasado estos niños y adolescentes.

\section{Familias expulsoras}

Muchas de las familias de los niños $y$ adolescentes en situación de calle fueron negativas para ellos $e$ influyeron en su condición de calle. Llamamos a estas familias expulsoras, pues por distintos factores (como la violencia física y verbal, una organización familiar deficiente o la situación de pobreza), expulsaron al niño o adolescente del espacio familiar, al espacio callejero.

\section{Situación de pobreza}

Para esta parte se han tomado en cuenta dos aspectos relacionados con la situación de pobreza, el primero: la incapacidad de cubrir las necesidades básicas, y el segundo: la vivienda y el barrio.

Con respecto al primero (la incapacidad de cubrir las necesidades básicas), se identificaron dos escenarios de calle. De acuerdo con Jorge Saavedra, director de CIMA, el primero se da como consecuencia de la ausencia de lospadres, quienes deben salir a trabajar por jornadas de tiempo largas, dejando a sus hijos en situación de abandono parcial. En ocasiones, cuando se trata de familias nucleares monoparentales, el niño o adolescente se queda en casa sin una figura de autoridad, lo que deja abierto el camino al espacio callejero.

Saavedra indica que existe un segundo escenario de calle, cuando los padres llevan a sus hijos (niños o adolescentes) a las calles para trabajar con ellos. Esta situación también se da por la situación de pobreza y la incapacidad de cubrir las necesidades básicas. Durante el trabajo de campo se encontró a niños y adolescentes trabajando en las calles con familiares, la mayoría de ellos como ambulantes. Dentro de los motivadores que llevaron a niños o adolescentes a la calle, se halló que el $43 \%$ se encontraba en este espacio por una motivación económica.
Una vez que el niño o adolescente empieza a frecuentar la calle, esta puede convertirse en una opción de sustento económico y emocional frente a un hogar donde sus necesidades básicas no son cubiertas y no existe una figura permanente de afecto y de control. Pedro, adolescente en proceso de rehabilitación, comenta que en la calle encontró el sustento económico del cual carecía en su hogar, este se presentó a través de su incorporación en una pandilla.

La vivienda y el barrio constituyen un segundo factor relacionado con la pobreza. De acuerdo con los especialistas Saavedra y Cabrera (asistenta social en Juan Pablo II), la mayoría de niños y adolescentes en situación de calle viven en muy malas condiciones físicas, que se ven empeoradas por los barrios en los que se encuentran, donde suele existir delincuencia.

En una oportunidad fuimos a la casa de uno de los niños para buscar a su familia y vimos varias cruces afuera $y$ pinturas de personas en las paredes. Los vecinos indicaban que ahí fallecían semanalmente y las familias ponían las cruces y pintaban sus rostros.

(Godoy, comunicación personal)

Asimismo, algunos adolescentes comentaron que los barrios en los que vivían eran inseguros. "Por mi zona, había gente que robaba con armas, había 'drogos' que andaban en moto y vendían drogas [...]. Asaltaban a las personas que caminaban por los callejones". (Adolescentes, centro de rehabilitación)

De esta forma, la pobreza de presenta a través de la incapacidad de cubrir las necesidades básicas, de viviendas que se encuentran en malas condiciones y de barrios peligrosos. Estos tres puntos (juntos o separados), pueden influir en la situación de calle de niños y adolescentes que están en busca de sustento económico y seguridad (la cual puede verse "compensada" con la pertenencia a pandillas).

\section{Organización familiar}

Un segundo aspecto de estudio dentro del tema "ambiente familiar" es la organización familiar. Para Manoel Torquato, presidente de 
la Campaña Nacional Crianza Nao e de Rua en Brasil, el círculo familiar más cercano del niño o adolescente cumple un rol fundamental en su situación de calle. Torquato hace referencia a la importancia no solo del padre y de la madre, sino de quien esté en mayor contacto con el niño o adolescente, ya sean sus tíos, abuelos u otro familiar.

El $33 \%$ de los niños y adolescentes en situación de calle, que fue parte de la investigación, proviene de familias nucleares monoparentales, es decir, donde viven con un solo padre. La mayor parte de familias nucleares monoparentales cuenta con la presencia de la madre (el $28 \%$ vive solo con su madre o con su madre y hermanos), un porcentaje bastante más bajo, cuenta con la figura del padre (el $5 \%$ vive solo con su padre o con su padre y hermanos).

Si se comparan estos resultados con las cifras de organización familiar a nivel nacional, en esta investigación el porcentaje de familias nucleares monoparentales es menor. El Observatorio Nacional de las Familias del MIMP señala que en Perú el $12 \%$ de las familias son nucleares monoparentales. (MIMP, sf, sp).

El siguiente gráfico muestra la organización familiar de los niños y adolescentes en situación de calle que fueron parte de la investigación:

\section{Organización familiar de los niños, niñas y adolescentes en situación de calle}

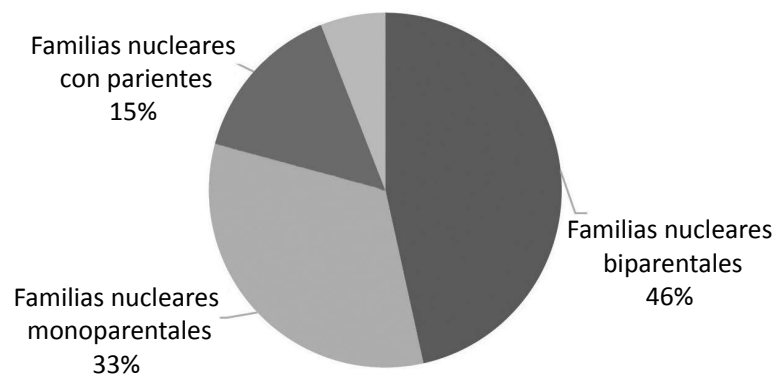

Familias nucleares biparentales Familias nucleares monoparentales

Familias nucleares con parientes $\square$ Familias con equivalentes familiares

Figura 1. Organización familiar de los niños, niñas y adolescentes en situación de calle.

Fuente: Vox Populi, 2017, p. 25, elaboración propia.
En el caso que se muestra a continuación, el menor de edad consumía drogas con sus amigos cuando sus familiares salían a trabajar, en algunas ocasiones evadía la asistencia al colegio:

En mi casa yo me quedaba solo, todos salían a trabajar. Mi cuñada salía a estudiar a las diez y de ahí yo me quedaba con los amigos hasta las once o doce; entonces ahí (me drogaba). [...] y luego, cuando yo estudiaba a la una en turno tarde y me iba al colegio, me echaba mis gotitas, a veces no entraba (me quedaba en la calle). (Adolescente, centro de rehabilitación)

De acuerdo con la psicóloga Flavia Centurión, la presencia de los padres es importante pues ellos son figuras de afecto y de autoridad. Según comenta, esta presencia es necesaria para que el menor de edad tenga límites en su comportamiento, sobre todo cuando está de por medio el bienestar de otros miembros de la sociedad.

\section{Violencia física y verbal}

Según los expertos entrevistados, muchos niños y adolescentes en situación de calle sufrieron de violencia física y verbal en sus hogares, este escenario genera la búsqueda de "seguridad" o "protección" por parte de los menores de edad en otros lados, fuera de su hogar. De acuerdo con su experiencia, en un centro de rehabilitación para adolescentes, Saavedra comenta que los menores en condición de calle generalmente se encuentran huyendo de una situación que les hace daño, como la violencia física.

Hablo de niños, niñas y adolescentes que en los primeros años han sentido la ausencia del amor y de la protección. Han sufrido la agresión física o el maltrato por parte de sus padres, de sus familiares y buscan refugiarse, buscan la seguridad o la protección en otros lados”. (Vox Populi, 2017, p. 29)

Godoy indica que la violencia física tiene su origen en la violencia verbal y que la mayoría de niños y adolescentes que han estado en 
situación de calle han sufrido de maltrato físico en sus hogares. En este punto coincide con Saavedra, comenta que la situación de violencia genera la búsqueda de un grupo de soporte.

Inicia con la violencia verbal. Muchos de ellos [los padres] son negligentes. Muchas veces por la cultura por omisión. Y bueno, maltratadores físicos, que es lo que en un $80 \%$ se ve acá mismo. Por un vacío los chicos van al internet, a la calle, a buscar un grupo, a las drogas, no es que ellos vayan de frente. (Godoy, comunicación personal) (Vox Populi, 2017, p. 28)

La especialista también señala que existe un vínculo entre la situación de pobreza y la violencia física. Los padres se frustran cuando no pueden cubrir las necesidades básicas de sus hijos, en oportunidades esta frustración se canaliza a través de la violencia verbal y física. De acuerdo con la especialista, el maltrato empieza con la violencia verbal hasta convertirse en violencia física, esto va escalando y se vuelve sistemático en el tiempo.

A los padres les falta ayuda para canalizar sus frustraciones. La más grande de las frustraciones es que no pueden darle lo básico. Y el niño siempre está "pero papá, necesito esto". Se ha visto muchas veces que los padres han infringido golpes de menos a más, al final, no habiendo un motivo aparente. Todo ha iniciado porque les han pedido las cosas básicas que no tenían. (Vox Populi, 2017, p. 31)

El 78\% de los niños y adolescentes en situación de calle encuestados afirma que en sus hogares existía violencia verbal (Vox Populi, 2017). De acuerdo con la ENARES 22016 , el 33\% de niños y adolescentes en Perú, ha sufrido de maltrato psicológico en los últimos doce meses, porcentaje que se encuentra por debajo del $78 \%$ de niños y adolescentes en situación de calle encuestado, que manifestó que en su hogar lo agredían verbalmente.

\section{Violencia verbal}

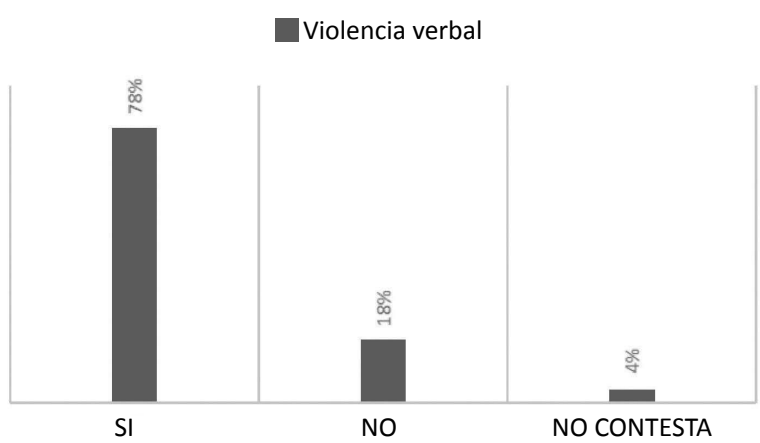

Figura 2. Violencia verbal en los hogares de los niños, niñas y adolescentes en situación de calle.

Fuente: Vox Populi, 2017, p. 29, elaboración propia.

En el caso de la violencia física, se trata del $48 \%$ de niños y adolescentes en situación de calle encuestados que declara que en sus hogares existe violencia física. Sin embargo, este porcentaje sería mayor por el sesgo existente en la pregunta. Aun así, esta cifra está por encima de los resultados de la ENDES ${ }^{3} 2015$, donde se señala que el $25 \%$ de los padres en Perú recurren a los castigos físicos como forma de reprimenda.

Además, la ENARES 2015 indica que el porcentaje que fue víctima de violencia física en los últimos doce meses es de $26.7 \%$, el cual también se encuentra por debajo de los resultados del presente estudio (INEI, 2016, s.p).

Pedro, adolescente en rehabilitación al cual se entrevistó para realizar la presente investigación, indica que su madre lo agredía física y verbalmente. La violencia verbal iba más allá de los gritos y consistía en frases hirientes hacia Pedro. "(Mi mamá) me gritaba y me golpeaba [...]. No sirves para nada, maldigo el momento en el que conocí a tu padre me decía. Bastardo me decía [...] No sentía nada porque ya estaba acostumbrado a los golpes... desde los tres años [...]. (Me pegaba) Con palo, con manguera" (Vox Populi, 2017, p. 33)

2 Encuesta Nacional sobre Relaciones Sociales realizada periódicamente por el Instituto Nacional de Estadística e Informática (INEI).

3 Encuesta Demográfica y de Salud Familiar realizada periódicamente por el INEI. 


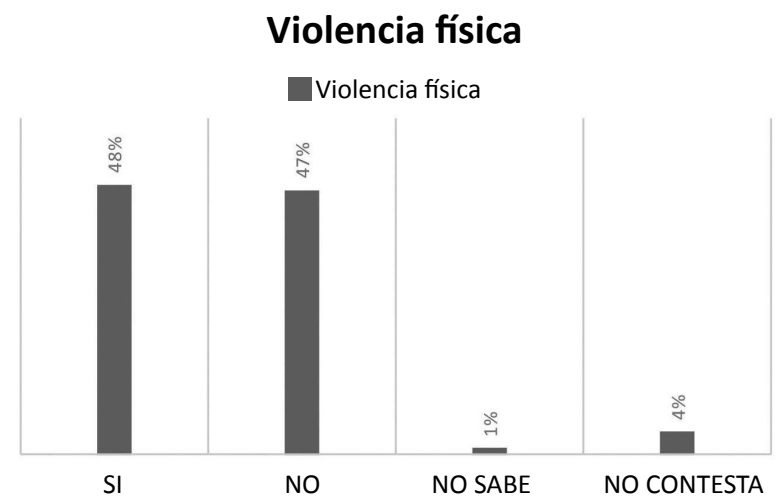

Figura 3. Violencia física en los hogares de los niños, niñas y adolescentes en situación.

Fuente: Vox Populi, 2017, p. 31, elaboración propia.

Con respecto al familiar agresor, el $29 \%$ de los niños y adolescentes en situación de calle encuestados indica que es solo su madre y el $21 \%$, solo su padre. Estos datos coinciden con lo hallado en las entrevistas en profundidad, donde los menores señalaron que era su madre quien ejercía violencia (física o verbal) en mayor medida.

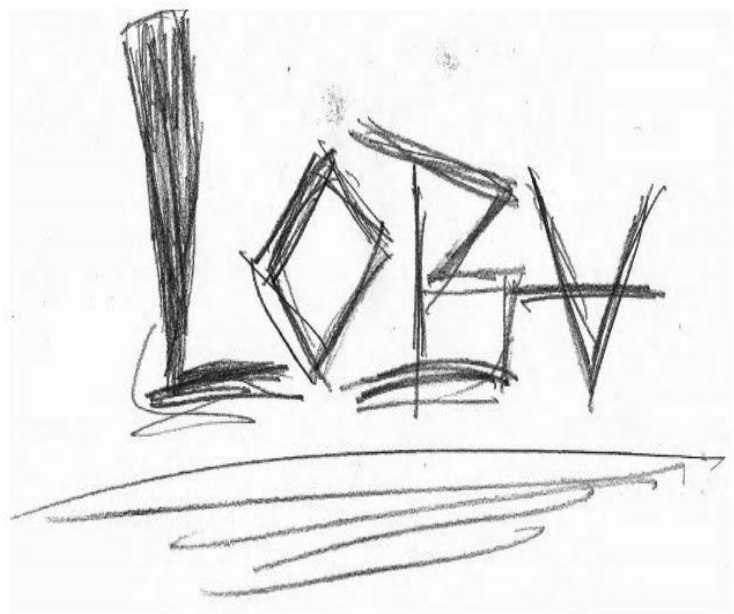

Figura 4. Dibujo de Pedro sobre su madre.

Fuente: Vox Populi, 2017, p. 42.

Asimismo, es importante tener en cuenta que el $18 \%$ afirmó que son otros familiares quienes ejercen violencia sobre ellos, pueden ser los tíos, abuelos, hermanos, etc. Y representan lo comentado por Torquato acerca de la importancia de la organización familiar que trasciende la presencia del padre y de la madre.

Además de la violencia de los padres hacia los hijos, también existe violencia entre los padres, donde según Centurión el padre suele ser el agresor y la madre la víctima, quien luego va a ejercer violencia física o verbal sobre el niño o adolescente.

Estas situaciones pueden terminar en tragedias y generar impactos muy negativos en los niños, como el hecho que narra Godoy.

Un papá fue asesinado por la familia de la mamá. [...]. Él había presenciado (el niño). La familia de ella lo había asesinado debido a que ella había recibido muchos maltratos por parte de él. La familia reaccionó, y en una situación la familia lo acuchilla y él fallece. A veces ellos (los niños) quieren dar lujos de detalles, lo tienen tan vívido (Vox Populi, 2017, p. 35)

La situación de violencia física y verbal de los padres hacia los hijos o entre los padres genera un ambiente negativo en el cual el niño o adolescente es dañado no solo física, sino, psicológicamente. Este entorno puede ser uno de los principales motivadores para la situación de calle que encuentran en este espacio una forma de evasión.

\section{Educación y espacio escolar}

En este tercer punto se estudió la situación de la educación y del espacio escolar de los niños y adolescentes en situación de calle que fueron parte de la investigación.

Existen dos grupos, quienes nunca fueron al colegio y quienes dejaron de asistir. Con relación a los niños y adolescentes que nunca fueron al colegio, el $49 \%$ indica que fue porque estaba en la calle, el $26 \%$ porque sus padres no lo llevaron y el $25 \%$ porque no quiso ir. (Vox Populi, 2017, p. 56)

Por otro lado, los motivos por los cuales los niños y adolescentes en situación de calle encuestados dejaron de ir al colegio fueron principalmente por rechazo al colegio $(45 \%)$ y por su situación económica (30\%). En menor medida por expulsión (6\%) e ingreso a un centro de rehabilitación o casa hogar (6\%). 


\section{Motivo por el que el niño o adolescente dejó de ir a colegio}

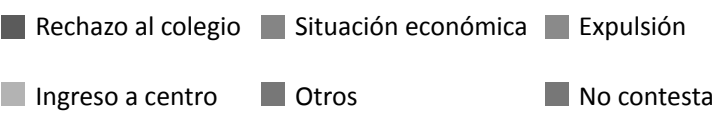

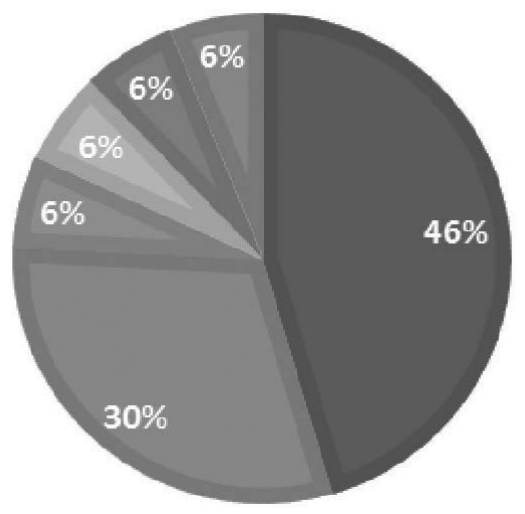

Figura 5. Motivo por el cual el niño o adolescente en situación de calle dejó de ir al colegio.

Vox Populi, 2017, p. 54, elaboración propia.

Asimismo, el $51 \%$ de los niños y adolescentes en situación de calle encuestados respondió que se sentía "regular/mal" en el colegio, mientras que el $48 \%$ respondió que se sentía "bien".

Ya que el $45 \%$ de los niños y adolescentes encuestados dejó de ir al colegio por una cuestión de rechazo, y el $51 \%$ dijo que se sentía "regular o mal" en este espacio de educación básica regular, el tema fue abordado en las entrevistas en profundidad y focus groups, donde se encontró que una de las principales razones de rechazo hacia el colegio es el bullying entre alumnos y el maltrato de profesores hacia alumnos.

Los adolescentes del centro de rehabilitación indicaron que preferían no ir al colegio porque los profesores ejercían maltrato físico y verbal contra ellos. "Cuando no entendías la profesora te pegaba, te gritaba y te pegaba con palo". (Adolescente, centro de rehabilitación) (Vox Populi, 2017, p. 59)

En la casa hogar los menores de edad expresaron que el colegio resultaba aburrido para ellos, pero además, su rechazo se debía a la "falta de justicia" por parte de los profesores. Según señalan, los profesores castigaban a quienes no les correspondía recibir un castigo.

\section{Calle}

La calle o el espacio callejero constituye un entorno lleno de peligros para un niño o adolescente. En este aspecto se investigó el motivo (económico o de evasión) para la situación de calle, la actividad realizada por los niños o adolescentes, la actividad realizada por sus conocidos, el pandillaje (la incorporación en pandillas, el consumo de drogas y las infracciones), y la valoración (positiva o negativa acerca del ámbito callejero).

\section{Motivador}

De acuerdo con los resultados de la investigación, existen dos motivaciones principales para la situación de calle de niños y adolescentes. La primera es económica, y corresponde a los menores de edad que se dedican al trabajo en calle. La segunda es de evasión, y corresponde a los niños y adolescentes que se encuentran haciendo otro tipo de actividades como "jugar", "jugar en cabinas", "socializar con amigos", etc. Actividades que se verán a continuación.

\section{Actividad}

Como se comentaba, las actividades son de dos tipos: económicas o de evasión. Las actividades económicas ejercidas por los niños y adolescentes en situación de calle encuestados, fueron las siguientes: venta ambulante (30\%), ayuda a familiares (14\%), lustrabotas (10\%), mendigar (4\%), jalador (3\%) y limpiar carros (2\%).

Las actividades de distracción o evasión son: estar con amigos (19\%), jugar en cabinas (20\%) y jugar (12\%).

Cabe resaltar que cada una de estas actividades no es exclusiva, los niños y adolescentes en situación de calle pueden realizar más de una actividad: por ejemplo, trabajar en la venta ambulante y jugar, o estar con los amigos y jugar en cabinas. 


\section{Actividad realizada por los niños o adolescentes en situación de calle}

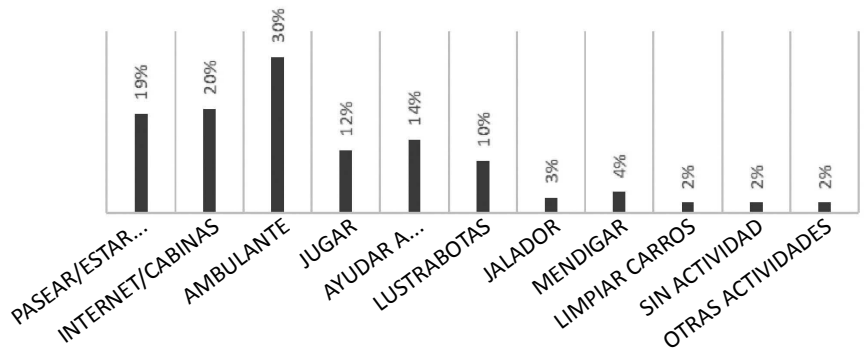

Figura 6. Actividad que el niño o adolescente realizaba con mayor frecuencia en la calle.

Fuente: Vox Populi, 2017, p. 74, elaboración propia.

Durante el trabajo de campo cuantitativo se registraron los casos de distintos niños y adolescentes realizando trabajo infantil en la calle. En uno de los casos, dos adolescentes trabajaban como lustrabotas desde las 06:00 hasta las 13:00 y luego desde las 16:00 hasta finalizar la noche. Utilizaban un intermedio para regresar a su vivienda y dar el almuerzo a sus hermanos menores. Otro caso bastante común fue el de niños y adolescentes realizando venta ambulante en distintas calles, junto con sus padres o familiares.

\section{Actividad que realizan en calle, los conocidos del niño o adolescente en situación de calle}

La calle es un espacio de riesgos, como se comentaba al inicio, muchos de estos riesgos provienen de otros actores presentes en las calles. El $54 \%$ de las personas que los niños y adolescentes en situación de calle, han conocido en la calle, es vendedor. El $32 \%$ se dedica a robos, el $28 \%$ pertenece a pandillas, el $28 \%$ es artista callejero y el $13 \%$ a la prostitución.

\section{Actividades a las que se dedican las personas que los niños y adolescentes en situación de calle conocen en la calle}

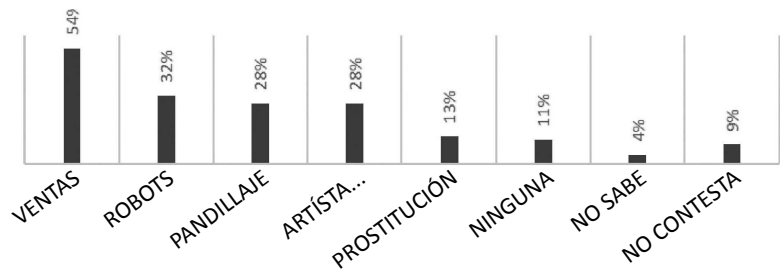

Figura 7. Actividades a las que se dedican las personas que los niños y adolescentes conocen en la calle.

Fuente: Vox Populi, 2017, p. 79, elaboración propia.

\section{Pandillaje}

El pandillaje es uno de los principales riesgos para un niño o adolescente que interactúa con la calle. Este riesgo se puede ver manifestado de dos formas: incorporarse en una pandilla, o verse afectados por el comportamiento de los miembros de las pandillas, quienes suelen actuar de forma violenta, cometer robos $y$ otros tipos de infracciones.

Primero es importante definir el concepto de pandilla, de acuerdo con los niños y adolescentes que formaron parte de la investigación, las pandillas son grupos de menores de edad, liderados por un adolescente o joven mayor que el resto de integrantes. Las principales actividades de las pandillas son los robos y el consumo de drogas ilegales y de alcohol. "Nuestra pandilla, lo que nos conformaba era poder fumar, tomar, robar, ir a las calles y hacer lo que nosotros queríamos con nuestras vidas. $L a$ mayoría del tiempo que pasábamos era robando" (Adolescente, centro de rehabilitación).

De acuerdo con los niños, niñas $y$ adolescentes entrevistados, la pandilla es una organización social del ámbito callejero encabezada por un líder, usualmente mayor que el resto de integrantes. En esta se manifiestan dos actividades fundamentales: el robo y el consumo de drogas y alcohol; también la asistencia a eventos (fiestas) y la agresión, reflejada en disputas entre los miembros de distintos grupos. (Vox Populi, 2017, p. 85)

El Código de los niños y adolescentes define a la "pandilla perniciosa" como el grupo de adolescentes entre 12 y 18 años que agrede o atenta contra la vida o la integridad de terceras personas y de los bienes de la sociedad. (Vox Populi, 2017, p. 84)

Según los resultados de la investigación, el $43 \%$ de los niños y adolescentes en situación de calle encuestados formó o forma parte de alguna pandilla. Estos resultados se lograron a través de la pregunta ¿Conoces a personas de alguna pandilla? con el objetivo de evitar el sesgo que contiene la pregunta al hacer referencia a un concepto negativo. 


\section{Consumo de drogas}

De la misma forma que se hizo la pregunta indirecta acerca de la pertenencia a pandillas, se hicieron las preguntas acerca del consumo de drogas. En este sentido, el $43 \%$ ha sido consumidor o consume alcohol.

El $24 \%$ probó alcohol por primera vez entre los 12 y 13 años, el $31 \%$, entre los 14 y 15 años.

Si se hace una división por grupos de edad, el $16 \%$ probó alcohol por primera vez en su niñez, entre los 6 y 11 años, y el $72 \%$ durante su adolescencia, entre los 12 y 17 años.

\section{Edad de inicio en el consumo del alcohol}

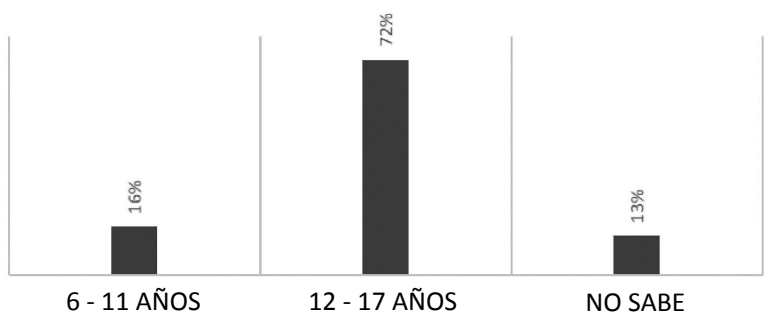

Figura 8. Edad de inicio en el consumo de alcohol de niños y adolescentes en situación de calle.

Fuente: Vox Populi, 2017, p. 97.

Con respecto a las drogas ilegales, se halló que el $40 \%$ de los niños y adolescentes en situación de calle que fueron parte de la investigación, había consumido algún tipo de droga ilegal.

Con relación a las edades, se da que el consumo de drogas se inicia a más temprana edad (el 25\% consumió por primera vez entre los 6 y 11 años, y el 56\%, entre los 12 y 17 años). Esto tiene coherencia con las actividades realizadas por las pandillas, donde los menores de edad manifestaron que una de las características del pandillaje es el consumo de drogas ilegales. Pedro indica que su pandilla consumía drogas ilegales en mayor medida que alcohol.

\section{Edad de inicio en el consumo del drogas ilegales}

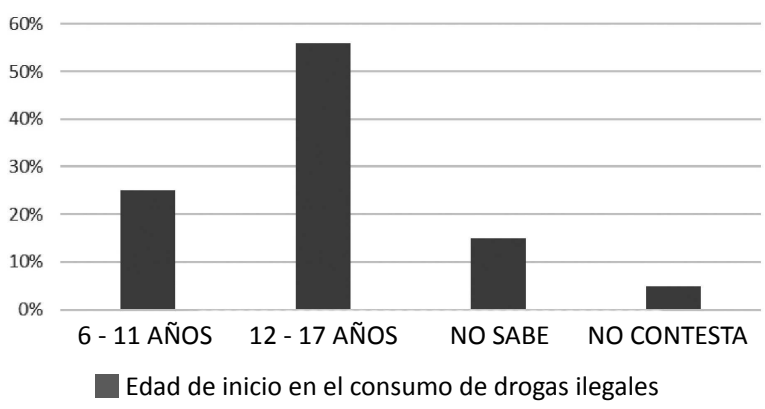

Figura 9. Edad de inicio en el consumo de drogas ilegales de niños y adolescente en situación de calle.

Fuente: Vox Populi, 2017, p. 102, elaboración propia.

Se encontró que una de las principales razones por las cuales niños y adolescentes se inician en el consumo de drogas a temprana edad es por la necesidad de evadir una situación que les hace daño. "Cuando tu papá y tu mamá se peleaban, tú sentías un dolor y ya consumías y se te hacía fácil olvidar, y te sentías más contento". (Adolescente, centro de rehabilitación)

Centurión y Godoy reafirman lo manifestado por el menor de edad, el consumo de drogas se da como una forma de escape ante una realidad que les duele. Pedro, por ejemplo, empezó a consumir drogas luego de que fuera asaltado por una pandilla del barrio en el que vivía, la cual también lo inició en el consumo. Para él, este era un momento en el cual podía olvidarse de todos los problemas que tenía.

Asimismo, se halló que la droga de mayor consumo es el terokal (59\%), seguida de la marihuana (50\%), la pasta básica de cocaína $(41 \%)$, el crack $(17 \%)$ y otras drogas $(11 \%)$. (Vox Populi, 2017, p. 105)

\section{Infracciones}

El pandillaje tiene como actividad fundamental el robo, pues este permite a sus integrantes comprar drogas. Según los testimonios recogidos, el principal destino de lo robado se destina a la compra de drogas, celulares, zapatillas y comida. David (exmiembro de una pandilla), señala que su pandilla robaba 
para comprar drogas, la comida pasaba a un segundo plano, para no gastar mucho dinero en su alimentación, ellos compartían los menús que compraban a la hora de almuerzo.

Según Cabrera, quienes roban y asaltan en mayor medida son los integrantes menores de las pandillas. Según comenta, los líderes están en busca de menores para que cometan las infracciones. "A los 12, 13 años el chico ya sale a la calle [...] comienzan con el robo y así se va iniciando el pandillaje. Siempre hay líderes de 18, 20 años, que captan a los niños en los barrios".

La búsqueda de niños y adolescentes para cometer robos no es casual. Los menores de edad son procesados bajo los artículos del Código de los Niños y Adolescentes, mas no del Código Penal, razón por la cual es conveniente para los líderes que sean menores de edad quienes cometen las infracciones (un menor de edad no es considerado un delincuente, sino, un infractor de la ley). Si el menor de edad aún no ha cumplido los 14, es un "mejor candidato" para ser quien realice robos y asaltos, a partir de los 14 años los adolescentes se pueden considerar infractores de la ley.

El asalto narrado por Pedro (14 años en ese momento), manifiesta cómo funcionan los asaltos, los cuales se dan en grupo y traen de por medio armas para amedrentar a quienes están siendo agredidos.

Era sábado pa domingo y estábamos yo, mi hermano, con mis amigos. Y mi amigo había llevado como tres desarmadores para ir a robar. Era un concierto de rock que quedaba en la playa donde yo estaba viviendo. Había una pareja que estaba un poco alejada del concierto y nosotros fuimos a robarles. La primera nos salió bien. La segunda no tan bien porque cuando fuimos a robarles, a mi amigo se le resbaló la navaja y se cortó la palma de la mano. De ahí ya no volví a la calle. (Vox Populi, 2017, p. 112)

Como se comentaba, las infracciones se dan por una motivación económica pero también por el carácter agresivo de las pandillas y la rivalidad existente entre ellas. Un ejemplo es el caso de un adolescente en un centro de rehabilitación que participó de una fuerte pelea entre pandillas. En esta ocasión, la agresión se dio en una fiesta semáforo, donde la pandilla del adolescente fue responsable de acuchillar a un miembro de otra pandilla. Ante la situación, el organizador del evento llamó a Serenazgo, el cual recibió sesenta soles por parte de los menores de edad para que no fueran llevados a la comisaría.

Otro es el caso narrado por un adolescente en un centro de rehabilitación cuyo hermano había pertenecido a una pandilla. "Cuando mi hermano tenía su pandilla, tenía un sable "grandazo", una vez agarraron a uno de otra pandilla por mi casa, lo golpearon y le metieron el sable en la espalda también le cortaron la mano". (Vox Populi, 2017, p. 113)

Cabe resaltar que el $19 \%$ de los niños y adolescentes en situación de calle encuestados reveló que tiene amigos en la cárcel. Este porcentaje se concentra en los adolescentes que se encuentran en centros de rehabilitación (32\%). (Vox Populi, 2017, p.115)

\section{Lo bueno y lo malo de la calle}

Para conocer qué valoración le han otorgado a la calle, se les preguntó qué era lo bueno y lo malo del espacio callejero. El $15 \%$ dijo que lo bueno es el trabajo, el $14 \%$, lo amigos/ hermanos/ enamorada, el $13 \%$, la libertad, el 8\%, el poder distraerse, el 8\%, el tener nuevas experiencias, el $6 \%$, ayudar a la casa (relacionado con el trabajo) y el $2 \%$, la ayuda para pagar los gastos del colegio (también relacionado con el trabajo).

Con respecto a lo "malo" de la calle, el $56 \%$ dijo que es la violencia/pandillaje/ delincuencia, el $12 \%$, el abuso de autoridad y el 3\%, los accidentes de tránsito. Lo malo de la calle está relacionado con sus experiencias, como se vio antes, el $40 \%$ pertenece o perteneció a pandillas, donde existe violencia y delincuencia (infracciones). 


\section{Lo malo de la calle para niños y adolescentes en situación de calle}

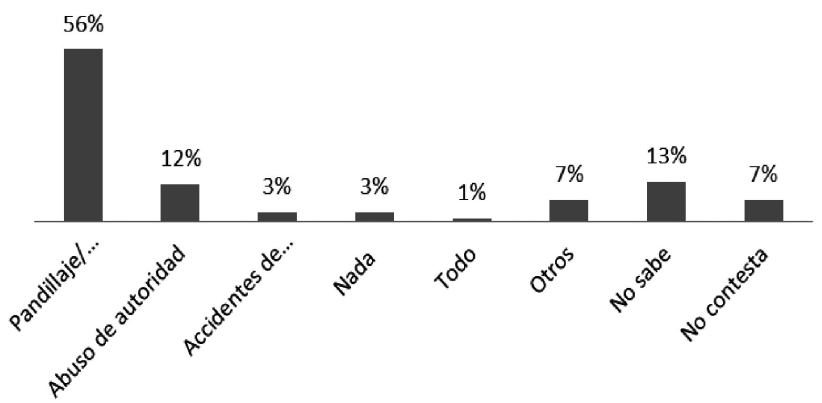

Figura 10. Lo malo de la calle según la percepción de los niños y adolescentes en situación de calle.

Fuente: Vox Populi, 2017, p.124, elaboración propia.

Para Pedro y David, lo malo de la calle es la delincuencia, las malas juntas y el consumo de drogas. David está preocupado por sus amigos, quienes siguen consumiendo drogas. Algunos ya son padres y están replicando los modelos aprendidos con sus hijos. David ha cambiado su perspectiva acerca del pandillaje desde que entró a un centro de rehabilitación, para él, el consumo de drogas y las infracciones no lo van a llevar a un futuro positivo.

\section{Sentido de pertenencia}

Se ha abordado el tema: sentido de pertenencia, como el nivel de identificación $y$ compromiso que tienen los niños $y$ adolescentes en situación de calle hacia el Perú, país en el cual nacieron y crecieron. (Vox Populi, 2017, p.145)

Para el 19\%, lo bueno del Perú es la gastronomía, para el $14 \%$, el turismo, para el $10 \%$ la naturaleza, para el 3\%, la amistad, para el $2 \%$, el trabajo, para el $1 \%$, la libertad, para el $2 \%$, nada y el $27 \%$ no sabe.

Lo malo del Perú, por otro lado, está relacionado con lo que ellos han experimentado en su corta vida y a través del espacio callejero. El $42 \%$ considera que lo malo del Perú es la delincuencia, la violencia y el pandillaje, conceptos que han aprendido en su experiencia en la calle.

\section{Lo malo del Perú}

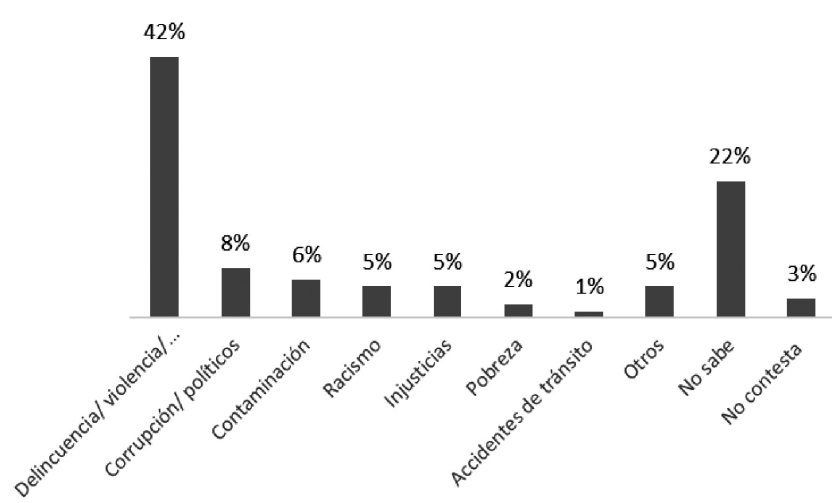

Figura 11. Lo malo del Perú según la percepción de los niños y adolescentes en situación de calle.

Fuente: Vox Populi, 2017, p.147, elaboración propia.

Para Pedro lo peor del Perú es la delincuencia. "Porque con la delincuencia hay muchas personas que sufren, que no regresan a su hogar. Si les robas y no te dan lo que quieres, los matas o los dejas malheridos y los tienen que llevar al hospital". (Vox Populi, 2017, p. 148)

Esto es lo que algunos adolescentes de un centro de rehabilitación dijeron acerca del Perú:

-Es un país de delincuencia, matanza, corrupción y mucha venta de drogas (Adolescente, centro de rehabilitación) (Vox Populi, 2017, p. 149)

- En Lurigancho hay fumones, maltratadores de las mujeres. Ahí están los rateros, hay puro grafiti y maleante, es cochino. La Molina es bonito, le cuidan, se respeta a la gente (Adolescente, Centro de rehabilitación) (Vox Populi, 2017, p. 150)

-Es un país lleno de delincuencia, drogadicción. Es un país vacío, como en blanco y negro, sin color, sin esperanza, es un país vacío. (Adolescente, centro de rehabilitación) (Vox Populi, 2017, p. 151)

Se puede entender que un grupo importante de los niños y adolescentes que están o han estado en condición de calle, que fueron parte de esta investigación, extienden el concepto que tienen acerca de su situación de calle, 
al resto del país, un lugar que califican como peligroso, violento, corrupto e injusto.

\section{Visión del futuro}

A pesar de que muchos niños y adolescentes en situación de calle provienen de familias expulsoras y han experimentado situaciones negativas en el espacio callejero, la percepción que tienen acerca de su futuro es positiva. El $86 \%$ considera que tendrá un buen futuro, el $11 \%$ que será malo y el $1 \%$ que será regular. (Vox Populi, 2017, p. 155)

David comenta que cree que tendrá un buen futuro pero que este será el resultado de mucho esfuerzo "Bien, pero también va a costar. Un camino donde hay rompe muelles, donde no te puedes quedar atrás". (Vox Populi, 2017, p. 156)

Con relación a los estudios superiores, el $70 \%$ señaló que le gustaría estudiar en la universidad, el $14 \%$ en un instituto técnico, al $6 \%$ no le gustaría estudiar y el $9 \%$ no sabe. Aunque se trata de una cifra alentadora, la realidad de muchos niños y adolescentes en situación de calle es que no terminan la educación básica regular necesaria para poder realizar estudios superiores. Además, el $92 \%$ manifestó que considera importante ir al colegio para su futuro.

\section{¿Te gustaría ir a la universidad, a un instituto técnico o no estudiar?}

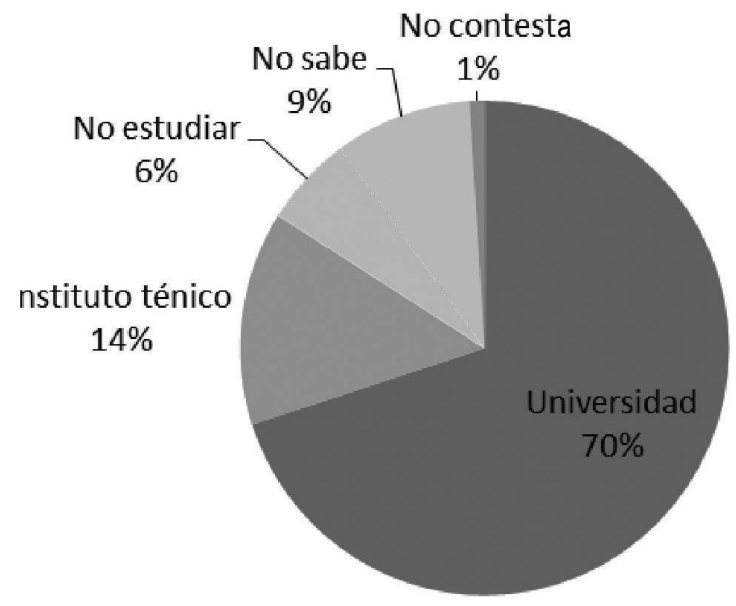

Figura 12. Deseo de tener formación superior de niños y adolescentes en situación de calle.

Fuente: Vox Populi, 2017, p. 163, elaboración propia.
Con respecto a su visión laboral, el porcentaje más alto fue el de los menores de edad que no tienen ninguna preferencia laboral-profesional para su futuro (29\%), sin embargo, el $16 \%$ quisiera ser militar, el 14\%, ingeniero, el 5\%, abogado, el $3 \%$, profesor, el $3 \%$, administrador, el $2 \%$, veterinario, el $2 \%$ diseñador gráfico y el $20 \%$ no sabe.

Pedro y David, por ejemplo, quisieran entrar a las fuerzas armadas. Pedro quiere ser un militar del ejército para demostrar a sus familiares y conocidos que ha superado la situación de calle y el pandillaje. David planea terminar la secundaria para inscribirse en la Marina. "Ahora que estoy en el centro, ya planeé mi futuro. Acabar mi secundaria y estudiar maquinaria pesada en la Marina, inscribirme en la Marina y ser marinero". (Vox Populi, 2017, p 166)

También se indagó acerca de la visión que tienen acerca de su futuro familiar. El $68 \%$ de los niños y adolescentes en situación de calle, que fueron parte de la investigación, quisiera formar una familia más adelante. El $61 \%$ planea tener su primer hijo entre los 23 y 30 años, sin embargo, este deseo se puede ver frustrado por los embarazos adolescentes no deseados, el $46 \%$ de las menores de edad que se encuentran en situación de calle, ya han tenido por lo menos un embarazo (la mayoría termina en abortos). (Vox Populi, 2017, p. 110)

\section{Edad a la cual le gustaría tener su primer hijo (para quienes respondieron que desean ser padres en el futuro)}

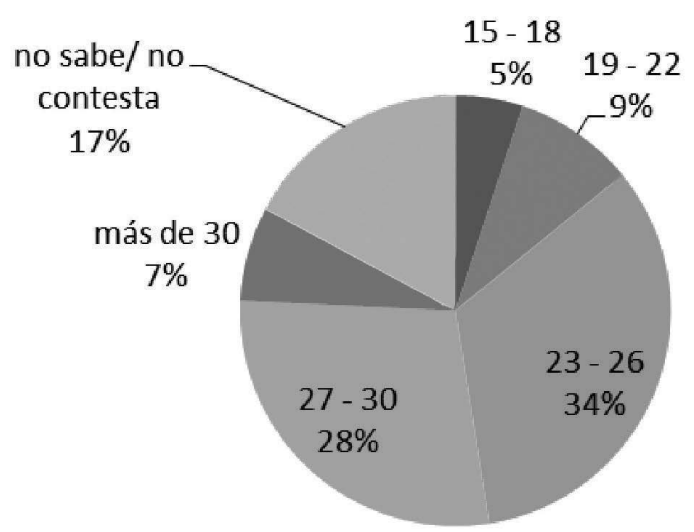

Figura 13. Edad en la cual los niños y adolescentes en situación de calle quisieran tener su primer hijo.

Fuente: Vox populi, 2017, p. 173, elaboración propia. 


\section{Conclusiones}

La familia de los niños y adolescentes que fueron parte de la investigación, influye en su condición de calle. Muchos de ellos viven o vivieron en hogares pobres, donde sus padres no podían cubrir sus necesidades básicas. Además, en muchos casos las viviendas se encuentran en malas condiciones $y$ en barrios peligrosos, donde existe delincuencia, consumo de drogas, violencia, etc.

La organización familiar de los menores de edad en cuestión se presenta como otro factor de incide en la situación de calle. El 33\% proviene de familias nucleares monoparentales, el 15\%, de familias nucleares con parientes (que no son los padres) y el $6 \%$, con equivalentes familiares (que no son familiares directos). La ausencia de los padres genera un vacío, pues ellos son una figura de afecto $y$ autoridad necesaria para el desarrollo de los niños. Cuando los padres salen a trabajar (sobre todo en las familias nucleares monoparentales), los menores se quedan solos por largos periodos, lo que facilita la salida a la calle, el inicio en el consumo de drogas y a incorporación en pandillas.

Un tercer aspecto es la violencia física y verbal ejercida por los padres hacia los hijos o entre los padres. El $78 \%$ de los niños y adolescentes que formaron parte de la investigación declaró que sufría de violencia verbal en su hogar, y el $48 \%$, de violencia física. La agresión sufrida suele ser sistemática y el nivel de violencia va escalando.

Con relación al colegio, los niños y adolescentes en situación de calle entrevistados manifestaron que el espacio escolar puede ser muy negativo, sobre todo porque algunos profesores ejercen violencia física y verbal hacía ellos. El $45 \%$ dejó el colegio por una cuestión de rechazo y el $30 \%$, por dificultades económicas. Asimismo, el $51 \%$ dijo haberse sentido mal o regular en el colegio.

Frente a dos situaciones negativas, fundamentales para el desarrollo de un niño $y$ adolescente, la calle se presenta como un falso aliado, porque ofrece sustento económico (a través del trabajo y del robo) y un soporte emocional (a través de un grupo de amigos con problemas similares). En oportunidades, el grupo de amigos de la calle es en realidad una pandilla. El $41 \%$ de los niños y adolescentes de la investigación, pertenece o perteneció a una pandilla.

La pandilla es una de las principales amenazas para niños y adolescentes en condición de calle. Se muestra como una alternativa positiva para quienes provienen de familias expulsoras. Sin embargo, la experiencia trae de por medio consumo de drogas ilegales, de alcohol y el inicio en la delincuencia (que de acuerdo con la ley, para los menores de edad es "infracción").

Las experiencias negativas por las cuales han pasado, han generado rechazo por parte de los niños y adolescentes en situación de calle, hacia su país, el Perú. Un lugar que califican como injusto, violento y lleno de delincuencia. Finalmente, el grupo de menores de edad en situación de calle que formó parte de la investigación, cree que tendrá un buen futuro $(86 \%)$. La mayoría sueña con ir a la universidad (70\%) y el $68 \%$ desea formar una familia. Los menores de edad que se entrevistaron en los centro de rehabilitación expresaron el deseo que tienen de reivindicarse frente a sus familiares y conocidos. 


\section{REFERENCIAS}

Adolescentes de CIMA (Comunicación personal, mayo 2016).

Benavente, Mariana (2017) Los niños de la calle. Lima, Perú: Vox Populi.

Centurión, Flavia (Comunicación personal, setiembre 2016).

Instituto Nacional de Estadística e Informática (2016) violencia física o psicológica hacia niños y adolescentes en los últimos 12 meses. Recuperado el 12 de septiembre de 2016 de: https://www. inei.gob.pe/prensa/noticias/ mas-del-40-de-las-ninas-y-ninos-fueron-victimas-de-violencia-fisicao-psicologicaen-los-ultimos-12-meses-9191/

Instituto Nacional de Estadística e Informática. (s.f.). Perú: Encuesta Demográfica y de Salud Familiar. Recuperado el 5 de julio, 2016 de: https://www.inei. gob.pe/media/MenuRecursivo/ publicaciones_digitales/Est/Lib1356/index.html

Ministerio de la Mujer y Poblaciones Vulnerables (2016) Observatorio Nacional de las Familias. Recuperado en febrero de 2017 de https:/observatoriodelasfamilias.mimp.gob.pe/

Yachay (2016) Los niños, niñas y adolescentes en situación de calle. Recuperado en octubre de 2016 de: https:/www.mimp.gob.pe/yachay/

Sinergia por la Infancia (2008) Perfil de niños, niñas y adolescentes en situación de calle. Lima, Perú: Voces para Latinoamérica

Godoy, Betty (Comunicación personal, mayo 2016).

Saavedra, Jorge (Comunicación personal, junio 2016).

Fecha de recepción: 02-10-17

Fecha de aceptación: 28-11-17 